\title{
Front Matter: Volume 8081
}

, "Front Matter: Volume 8081," Proc. SPIE 8081, Photomask and NextGeneration Lithography Mask Technology XVIII, 808101 (6 July 2011); doi: 10.1117/12.899897

SPIE. Event: Photomask and NGL Mask Technology XVIII, 2011, Yokohama, Japan 


\title{
PROCEEDINGS OF SPIE
}

\section{Photomask and Next-Generation Lithography Mask Technology XVIII}

\author{
Toshio Konishi \\ Editor
}

\section{3-15 April 2011}

Yokohama, Japan

\section{Sponsored by}

PMJ Photomask Japan

BACUS

SPIE

Published by

SPIE

This conference was canceled due to the earthquake off the northeast coast of Japan in March 2011. This volume includes some of the papers that were scheduled to be presented at the conference.

Volume 8081

Proceedings of SPIE, 0277-786X, v. 8081 
The papers included in this volume were part of the technical conference cited on the cover and title page. Papers were selected and subject to review by the editors and conference program committee. Some conference presentations may not be available for publication. The papers published in these proceedings reflect the work and thoughts of the authors and are published herein as submitted. The publisher is not responsible for the validity of the information or for any outcomes resulting from reliance thereon.

Please use the following format to cite material from this book:

Author(s), "Title of Paper," in Photomask and Next-Generation Lithography Mask Technology XVIII, edited by Toshio Konishi, Proceedings of SPIE Vol. 8081 (SPIE, Bellingham, WA, 2011) Article CID Number.

ISSN 0277-786X

ISBN 9780819486738

Published by

SPIE

P.O. Box 10, Bellingham, Washington 98227-0010 USA

Telephone +1 3606763290 (Pacific Time) · Fax +1 3606471445

SPIE.org

Copyright (C) 2011, Society of Photo-Optical Instrumentation Engineers

Copying of material in this book for internal or personal use, or for the internal or personal use of specific clients, beyond the fair use provisions granted by the U.S. Copyright Law is authorized by SPIE subject to payment of copying fees. The Transactional Reporting Service base fee for this volume is $\$ 18.00$ per article (or portion thereof), which should be paid directly to the Copyright Clearance Center (CCC), 222 Rosewood Drive, Danvers, MA 01923. Payment may also be made electronically through CCC Online at copyright.com. Other copying for republication, resale, advertising or promotion, or any form of systematic or multiple reproduction of any material in this book is prohibited except with permission in writing from the publisher. The CCC fee code is 0277-786X/11/ \$18.00.

Printed in the United States of America.

Publication of record for individual papers is online in the SPIE Digital Library.

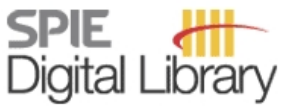

SPIEDigitalLibrary.org

Paper Numbering: Proceedings of SPIE follow an e-First publication model, with papers published first online and then in print and on CD-ROM. Papers are published as they are submitted and meet publication criteria. A unique, consistent, permanent citation identifier (CID) number is assigned to each article at the time of the first publication. Utilization of CIDs allows articles to be fully citable as soon as they are published online, and connects the same identifier to all online, print, and electronic versions of the publication. SPIE uses a six-digit CID article numbering system in which:

- The first four digits correspond to the SPIE volume number.

- The last two digits indicate publication order within the volume using a Base 36 numbering system employing both numerals and letters. These two-number sets start with 00, 01, 02, 03, 04, $05,06,07,08,09,0 A, 0 B \ldots 0 Z$, followed by 10-1Z, 20-2Z, etc.

The CID number appears on each page of the manuscript. The complete citation is used on the first page, and an abbreviated version on subsequent pages. Numbers in the index correspond to the last two digits of the six-digit CID number. 


\section{Contents}

vii Conference Committees

\section{MATERIALS AND PROCESS I}

808102 Mask blank material optimization impact on leading-edge ArF lithography [8081-44] K. Mesuda, H. Watanabe, K. Hayano, E. Tsujimoto, H. Takamizawa, Dai Nippon Printing Co., Ltd. (Japan); T. Ohhashi, N. Sakasai, S. Kudo, T. Matsuyama, Nikon Corp. (Japan)

\section{FPD PHOTOMASKS}

808105 Growth mechanism and inhibition technologies of a contamination on the surface of photomask for longtime LCD-TFT lithography process [8081-20]

M. Murai, M. Tsuchiya, H. Shinchi, T. Hirano, Hoya Corp. (Japan); S. Kitajima, Y. Kaneko,

T. Kimoto, S. Takayama, Asahi-Kasei E-materials Corp. (Japan)

808106 Particle free pellicle mounting inside of the large size photomask inspection system [8081-41]

M. Yonezawa, S. Matsumoto, D. Zhang, K. Hashimoto, Lasertec Corp. (Japan)

\section{MATERIALS AND PROCESS II}

808107 Improvement of polymer type EB resist sensitivity and line edge roughness [8081-22] M. Otani, H. Asada, Yamaguchi Univ. (Japan); H. Tsunoda, M. Kunitake, Kumamoto Univ. (Japan); T. Ishizaki, EMF Japan Inc. (Japan); R. Miyagawa, LLC Gluon Lab. (Japan)

$80810822 \mathrm{~nm}$ node ArF lithography performance improvement by utilizing mask 3D topography: controlled sidewall angle [8081-45]

H. Watanabe, K. Mesuda, K. Hayano, E. Tsujimoto, H. Takamizawa, Dai Nippon Printing Co., Ltd. (Japan); T. Ohhashi, N. Sakasai, S. Kudo, T. Matsuyama, Nikon Corp. (Japan)

\section{PHOTOMASK WRITING TOOLS AND TECHNOLOGIES}

808109 Advancing the charging effect correction with time-dependent discharging model [8081-40]

N. Nakayamada, T. Kamikubo, H. Anze, S. Tamamushi, NuFlare Technology, Inc. (Japan)

8081 OA EB resolution capability with CP exposure [8081-07]

M. Kurokawa, H. Isobe, K. Abe, Y. Oae, A. Yamada, Advantest Corp. (Japan); S. Narukawa, M. Ishikawa, H. Fujita, M. Hoga, N. Hayashi, Dai Nippon Printing Co., Ltd. (Japan) 
INSPECTION TOOLS AND TECHNOLOGIES I

8081 OD Practical mask inspection system with printability and pattern priority verification [8081-30]

H. Tsuchiya, F. Ozaki, K. Takahara, T. Inove, N. Kikuiri, NuFlare Technology, Inc. (Japan)

METROLOGY TOOLS AND TECHNOLOGIES I

8081 OG New CD-SEM metrology method for the side wall angle measurement using multiple detectors [8081-28]

H. Fukaya, T. Murakawa, S. Shida, M. Kuribara, T. Iwai, J. Matsumoto, T. Nakamura, Advantest Corp. (Japan); H. Hakii, I. Yonekura, M. Kawashita, Y. Nishiyama, K. Tanaka, Y. Kikuchi, Toppan Printing Co., Ltd. (Japan)

\section{LITHOGRAPHY I}

$8081 \mathrm{OH} \quad$ MRC optimization for EUV high NA imaging for the 32-nm HP technology node [8081-05] S.-E. Tseng, A. Chen, ASML Taiwan, Ltd. (Taiwan)

MDP, EDA, AND DFM

8081 ol Mask data processing technique using GPU for reducing computer cost [8081-31]

R. Tsujimura, K. Ogino, H. Hoshino, S. Satoh, Fujitsu Semiconductor Ltd. (Japan); K. Morishita, S. Yoshikawa, Fujitsu VLSI Ltd. (Japan); H. Futatsuya, T. Chijimatsu, S. Asai, S. Yamauchi,

T. Okada, N. Ishiwata, M. Miyajima, Fujitsu Semiconductor Ltd. (Japan)

\section{INSPECTION TOOLS AND TECHNOLOGIES II}

8081 OK The coherent EUV scatterometry microscope for actinic mask inspection and metrology [8081-04]

T. Harada, M. Nakasuji, T. Kimura, Univ. of Hyogo (Japan) and RIKEN (Japan); Y. Nagata, Japan Science and Technology Agency (Japan) and RIKEN (Japan); T. Watanabe,

H. Kinoshita, Univ. of Hyogo (Japan) and RIKEN (Japan)

\section{METROLOGY TOOLS AND TECHNOLOGIES II}

8081 OL In-die job automation for PROVE [8081-32]

R. J. Lesnick, Jr., S. Kim, Synopsys, Inc. (United States); M. Waechter, D. Seidel, A. Mueller,

D. Beyer, Carl Zeiss SMS GmbH (Germany) 
8081 ON Collaborative research on emerging technologies and design [8081-29]

A. R. Neureuther, J. Rubinstein, M. Miller, K. Yamazoe, E. Chin, C. Levy, L. Wang, N. Xu, C. Spanos, K. Qian, K. Poolla, J. Ghan, A. Subramanian, T.-J. King Liu, X. Sun, Univ. of California, Berkeley (United States); K. Jeong, Univ. of California, San Diego (United States); P. Gupta, A. Kaqalwalla, R. Ghaida, T.-B. Chan, Univ. of California, Los Angeles (United States)

$808100 \quad$ Evaluation of process variations on OPC model predictions [8081-18]

J. Oberschmidt, IBM Microelectronics (United States); S. Barai, IBM Microelectronics (India);

T. Desouky, Mentor Graphics Egypt (Egypt); O. P. Jaiswal, IBM Microelectronics (India);

A. Padmawar, Indian Institute of Technology Bombay (India); R. M. Pusuluri, IBM Microelectronics (India)

8081 OP New yield-aware mask strategies [8081-27]

K. Jeong, A. B. Kahng, Univ. of California, San Diego (United States); C. J. Progler, Photronics, Inc. (United States)

$80810 Q \quad$ Defect printability of advanced binary film photomask [8081-34]

M. Naka, S. Yamaguchi, K. Morishita, S. Kanamitsu, R. Yoshikawa, H. Mashita, T. Hirano, Toshiba Corp. (Japan)

8081 OR Role of ellipsometry in DPT process characterization and impact of performance for contact holes [8081-36]

I. Kamohara, Nihon Synopsys G.K. (Japan); V. Domnenko, Synopsys, Inc. (Russian

Federation); A. Philippou, Synopsys GmbH (Germany)

MDP

8081 OS Elimination of lithographic hotspots which have been waived by means of pattern matching [8081-17]

A. Chaudhary, IBM Corp. (India); P. Bouchard, K. Dave, IBM Corp. (United States);

T. Desouky, Mentor Graphics Egypt (Egypt); K. Moamen, M. Simmons, Mentor Graphics

Corp. (United States)

8081 OT Efficient method for SRAF rule determination [8081-35]

R. M. V. M. Pusuluri, P. Y. Bashaboina, IBM Microelectronics (India); J. M. Oberschmidt, IBM

Microelectronics (United States)

8081 OU Model-based mask data preparation (MB-MDP) for ArF and EUV mask process correction [8081-23]

K. Hagiwara, D2S, KK (Japan); I. Bork, A. Fujimura, D2S, Inc. (United States)

\section{MASK REPAIR}

8081 OV RegC: a new registration control process for photomasks after pattern generation [8081-39]

E. Graitzer, G. Ben-Zvi, A. Cohen, D. Vladimir, D. Avizemer, Carl Zeiss SMS Ltd. (Israel) 


\section{MASK DEGRADATION}

8081 OW Binary 193nm photomasks aging phenomenon study [8081-25]

F. Dufaye, STMicroelectronics Crolles (France); L. Sartelli, C. Pogliani, DNP Photomask Europe (Italy); S. Gough, F. Sundermann, STMicroelectronics Crolles (France); H. Miyashita, Y. Hidenori, DNP Photomask Europe (Italy); N. Charras, C. Brochard, N. Thivolle, STMicroelectronics Crolles (France)

8081 OX 193-nm radiation durability study of MoSi binary mask and resulting lithographic performance [8081-26]

I. Servin, J. Belledent, L. Pain, CEA-LETI MINATEC (France); B. Connolly, Toppan Photomasks, Inc. (Germany); M. Sczyrba, Advanced Mask Technology Ctr. GmbH Co. KG (Germany);

M. Lamantia, Toppan Photomasks Inc. (United States)

Author Index 


\title{
Conference Committees
}

\author{
Symposium Chair
}

Toshiyuki Horiuchi, Tokyo Denki University (Japan)

Vice Chair

Masato Shibuya, Tokyo Polytechnic University (Japan)

Advisory Committee Chair

Yasuo Tarui, Tokyo University of Agriculture and Technology (Japan)

Members

Masanori Komuro, New Energy and Industrial Technology

Development Organization (Japan)

Masatoshi Migitaka, Toyota Technological Institute (Japan)

Masao Otaki, Toppan Printing Company, Ltd. (Japan)

Norio Saito, Nippon Institute of Technology (Japan)

Yoshio Tanaka, Luminescent Technologies Inc. (Japan)

Organizing Committee Chair

Toshiyuki Horiuchi, Tokyo Denki University (Japan)

Vice Chair

Masato Shibuya, Tokyo Polytechnic University (Japan)

Members

Uwe Behringer, UBC Microelectronics (Germany)

Parkson Chen, Taiwan Mask Corporation (Taiwan)

Han-ku Cho, Samsung Electronics Company, Ltd. (Korea, Republic of)

Brian J. Grenon, Grenon Consulting, Inc. (United States)

Hideaki Hamada, HTL Company Japan Ltd. (Japan)

Naoya Hayashi, Dai Nippon Printing Company, Ltd. (Japan)

Morihisa Hoga, Dai Nippon Printing Company, Ltd. (Japan)

Eiichi Hoshino, Nikon Corporation (Japan)

Greg Hughes, International SEMATECH (United States)

Hiroichi Kawahira, Sony Corporation (Japan)

Toshio Konishi, Toppan Printing Company, Ltd. (Japan)

Yuichi Matsuzawa, Toppan Printing Company, Ltd. (Japan)

Warren Montgomery, SEMATECH (United States)

Ichiro Mori, Semiconductor Leading Edge Technologies, Inc. (Japan) 
Osamu Nagarekawa, HOYA Corporation (Japan)

Kenichiro Okuda, SEMI Japan (Japan)

Yoshiki Suzuki, KLA-Tencor Japan Ltd. (Japan)

Tadahiro Takigawa, Brion Technologies K.K. (Japan)

Koichiro Tsujita, Canon Inc. (Japan)

Wataru Wakamiya, JEOL Ltd. (Japan)

Anto Yasaka, SII NanoTechnology Inc. (Japan)

Nobuyuki Yoshioka, Renesas Electronics Corporation (Japan)

Steering Committee Chair

Morihisa Hoga, Dai Nippon Printing Company, Ltd. (Japan)

Vice Chairs

Hiroaki Morimoto, Toppan Printing Company, Ltd. (Japan)

Tsuneo Terasawa, Semiconductor Leading Edge Technologies, Inc. (Japan)

Members

Takayuki Abe, NuFlare Technology Inc. (Japan)

Naoya Hayashi, Dai Nippon Printing Company, Ltd. (Japan)

Kunihiro Hosono, Renesas Electronics Corporation (Japan)

Naoyuki Ishiwata, Fujitsu Semiconductor Ltd. (Japan)

Ichiro Kagami, Sony Corporation (Japan)

Kokoro Kato, SII NanoTechnology Inc. (Japan)

Toshio Konishi, Toppan Printing Company, Ltd. (Japan)

Hiroshi Mohri, Dai Nippon Printing Company, Ltd. (Japan)

Takeshi Nakajima, KLA-Tencor Japan Ltd. (Japan)

Masatoshi Oda, NTT Advanced Technology (Japan)

Yasushi Ohkubo, HOYA Corporation (Japan)

Hiroyuki Shigemura, Semiconductor Leading Edge Technologies, Inc. (Japan)

Toshiyuki Takahashi, JEOL Ltd. (Japan)

Kiwamu Takehisa, Lasertec Corporation (Japan)

Hiroyoshi Tanabe, Intel K.K. (Japan)

Hidehiro Watanabe, Toshiba Corporation (Japan)

Program Committee Chair

Toshio Konishi, Toppan Printing Company, Ltd. (Japan)

Vice Chairs

Ichiro Kagami, Sony Corporation (Japan)

Kokoro Kato, SII NanoTechnology Inc. (Japan)

Hiroshi Mohri, Dai Nippon Printing Company, Ltd. (Japan) 
Shinji Akima, Toppan Printing Company, Ltd. (Japan)

Akihiko Ando, Renesas Electronics Corporation (Japan)

Jeff Farnsworth, Intel Corporation (United States)

Thomas B. Faure, IBM Corporation (United States)

Shigeru Hirukawa, Nikon Corporation (Japan)

Koji Hosono, Fujitsu Semiconductor Ltd. (Japan)

Gregg Inderhees, KLA-Tencor Corporation (United States)

Curt A. Jackson, Toppan Photomasks, Inc. (United States)

Byung-Gook Kim, Samsung Electronics Company, Ltd. (Korea, Republic of)

Koichi Kuhara, HOYA Corporation (Japan)

John Lin, Taiwan Semiconductor Manufacturing Company, Ltd. (Taiwan)

Mark Ma, Photronics, Inc. (United States)

Koji Murano, Toshiba Corporation (Japan)

Yoshikazu Nagamura, Renesas Electronics Corporation (Japan)

Yoshinori Nagaoka, KLA-Tencor Japan Ltd. (Japan)

Yasutoshi Nakagawa, JEOL Ltd. (Japan)

Noriaki Nakayamada, NuFlare Technology Inc. (Japan)

Nobuyuki Nishiguchi, Semiconductor Technology Academic

Research Center (Japan)

Yuji Nonami, Panasonic Corporation (Japan)

Steffen Schulz, Mentor Graphics Corporation (United States)

Yasunari Sohda, Hitachi, Ltd. (Japan)

Osamu Suga, Semiconductor Leading Edge Technologies, Inc. (Japan)

Minoru Sugawara, Sony Corporation (Japan)

Koji Takagi, Applied Materials Japan, Inc. (Japan)

Kiwamu Takehisa, Lasertec Corporation (Japan)

Tatsuya Tomita, Dai Nippon Printing Company, Ltd. (Japan)

Yoichi Usui, HOYA Corporation (Japan)

Kenji Yamazoe, Canon Inc. (Japan)

Session Chairs

Invited Session

Toshio Konishi, Toppan Printing Company, Ltd.

Uwe Behringer, UBC Microelectronics (Germany)

Materials and Process

Thomas B. Faure, IBM Corporation (United States)

Koji Murano, Toshiba Corporation (Japan)

Special Session: FPD Photomasks

Ichiro Kagami, Sony Corporation (Japan)

Toshio Konishi, Toppan Printing Company, Ltd. (Japan) 
Writing Technologies

Yasutoshi Nakagawa, JEOL Ltd. (Japan)

Curt A. Jackson, Toppan Photomasks, Inc. (United States)

\author{
EUV Masks \\ Minoru Sugawara, Sony Corporation (Japan) \\ Kurt Kimmel, SEMATECH (United States) \\ Inspection Tools and Technologies \\ Gregg Inderhees, KLA-Tencor Corporation (United States) \\ Wolf Staud, Applied Materials (United States) \\ Metrology Tools and Technologies \\ Yoshinori Nagaoka, KLA-Tencor Japan Ltd. (Japan) \\ Yasunari Sohda, Hitachi, Ltd. (Japan) \\ OPC and Mask-related Lithography \\ Kenji Yamazoe, Canon Inc. (Japan) \\ Steffen Schulze, Mentor Graphics Corporation (United States)
}

DFM, EDA, and MDP

Kokoro Kato, SII NanoTechnology Inc. (Japan)

Nobuyuki Nishiguchi, Semiconductor Technology Academic Research Center (Japan)

NIL

Hiroshi Mohri, Dai Nippon Printing Company, Ltd. (Japan)

Akihiko Ando, Renesas Electronics Corporation (Japan)

Byung-Gook Kim, Samsung Electronics Company, Ltd. (Korea, Republic of)

Mask Repair

Hiroshi Mohri, Dai Nippon Printing Company, Ltd. (Japan)

Akihiko Ando, Renesas Electronics Corporation (Japan)

Byung-Gook Kim, Samsung Electronics Company, Ltd. (Korea, Republic of)

Mask Degradation

Hiroshi Mohri, Dai Nippon Printing Company, Ltd. (Japan)

Akihiko Ando, Renesas Electronics Corporation (Japan)

Byung-Gook Kim, Samsung Electronics Company, Ltd. (Korea, Republic of) 